\title{
Speech therapy for compensatory articulations and velopharyngeal function: a case report
}

\author{
Nachale Helen Maciel BISPO ${ }^{1}$, Melina Evangelista WHITAKER ${ }^{2}$, Homero Carneiro AFERRI ${ }^{3}$, Josiane Denardi Alves \\ NEVES ${ }^{4}$, Jeniffer de Cássia Rillo DUTKA ${ }^{5}$, Maria Inês PEGORARO-KROOK ${ }^{6}$

\begin{abstract}
1- Graduate student, Department of Speach-Patology and Audiology, Bauru School of Dentistry, University of São Paulo, Bauru, SP, Brazil.
2- PhD, Speech-Language Pathologist, Palatal Prosthesis Department, Hospital for Rehabilitation of Craniofacial Anomalies, University of São Paulo, Bauru, $\mathrm{SP}$, Brazil.

3- DDS, Palatal Prosthesis Department, Hospital for Rehabilitation of Craniofacial Anomalies, University of São Paulo, Bauru, SP, Brazil.

4- Speech-Language Pathologist, Palatal Prosthesis Department, Hospital for Rehabilitation of Craniofacial Anomalies, University of São Paulo, Bauru, SP, Brazil. 5- PhD, Communication Sciences and Disorders, Speech-Language Pathologist, Post Graduate Program at Hospital for Rehabilitation of Craniofacial Anomalies, University of São Paulo, Bauru, SP, Brazil.

6- PhD, Human Communication Disorders, Full Professor, Department of Speach-Patology and Audiology, Bauru School of Dentistry, University of São Paulo, Bauru, SP, Brazil.
\end{abstract}

Corresponding address: Melina Evangelista Whitaker - Rua Dr. Antonio Prudente, 4-152 - Jardim Estoril II - Bauru - SP - 17016-010 - Phone: (14) 32358096 - e-mail: mirian3m@uol.com.br

Received: July 26, 2010 - Accepted: August 16, 2011

\section{ABSTRACT}

$T_{6}^{\text {he }}$ he objective of this study was to describe the process of intensive speech therapy for a 6 -year-old child using compensatory articulations while presenting with velopharyngeal insufficiency (VPI) and a history of cleft lip and palate. The correction of VPI was temporarily done with a pharyngeal obturator since the child presented with very little movement of the pharyngeal walls during speech, compromising the outcome of a possible pharyngeal flap procedure (pharyngoplasty). The program of intensive speech therapy involved 3 phases, each for duration of 2 weeks incorporating 2 daily sessions of 50 minutes of therapy. A total of 60 sessions of intervention were done with the initial goal of eliminating the use of compensatory articulations. Evaluation before the program indicated the use of coproductions (coarticulations) of voiceless plosive and fricative sounds with glottal stops (simultaneous production of 2 places of productions), along with weak intraoral pressure and hypernasality, all compromising speech intelligibility. To address place of articulation, strategies to increase intraoral air pressure were used along with visual, auditory and tactile feedback, emphasizing the therapy target and the air pressure and airflow during plosive and fricative sound productions. After the first two phases of the program, oral place of articulation of the targets were achieved consistently. During the third phase, velopharyngeal closure during speech was systematically addressed using a bulb reduction program with the objective of achieving velopharyngeal closure during speech consistently. After the intensive speech therapy program involving the use of a pharyngeal obturator, we observed absence of hypernasality and compensatory articulation with improved speech intelligibility.

Key Words: Cleft palate. Children. Speech bulb. Speech therapy.

\section{INTRODUCTION}

Among the areas of Dentistry, Odontopediatrics, Orthodontics and Maxillofacial prosthesis are closely related with speech pathology. The prosthodontist and his partner, the prosthetic technician, are involved with the speech pathologist, particularly during treatment of oncologic and neurologic cases, as well as during management of craniofacial anomalies like cleft palate.

Cleft lip and palate (CLP) is a common congenital anomaly, occurring at a rate of 1:650 live births in Brazil $^{11}$. Rehabilitation of CLP in this country usually starts with primary surgeries to correct the cleft lip (cheiloplasty) and cleft palate (palatoplasty), performed most often during the first months and first year of life, respectively ${ }^{3}$. After the anatomic 
correction of the cleft, we may find inadequacies, such as the velopharyngeal dysfunction (VPD), a condition associated with communication and feeding disorders.

VPD is characterized by inadequate velopharyngeal functioning resulting in unwanted communication between oral and nasal cavities during production of oral speech sounds and during sucking and deglutition, also affecting middle ear function. Particularly in relation to speech, signs of VPD can include hypernasality, nasal air escape, weak intraoral air pressure during oral sound production, and use of atypical place of production (Compensatory Articulation - CA $)^{2,3,6}$.

When VPD is related to an insufficiency of tissue (for example, an absence of musculus uvulae or a short or hypoplastic palate) or to an increase in nasopharyngeal space (for example, a deep nasopharynx), a condition known as velopharyngeal insufficiency (VPI), the treatment requires a physical procedure (surgical or prosthetic) to establish a physical structure which allows for adequate velopharyngeal functioning. VPD can also be the result of velopharyngeal incompetency. In this case the physical/anatomical structure is sufficient to allow for adequate functioning, however, the functioning does not occur. Incompetency can be the result of neuro-sensory-motor alterations (paralysis or paresis) or even the result of incorrect learning of velopharyngeal functioning for speech production. Finally, VPD can be caused by an insufficiency combined with an incompetency.

Treatment of insufficiency may involve a secondary surgical procedure or a pharyngeal obturator. The obturator is made by the dentist in partnership with the speech pathologist and consists of a removable device with an anterior portion (acrylic plate with clips for retention), an intermediate portion (connecting the anterior portion to the bulb) and a pharyngeal bulb (the portion shaped to occupy the velopharyngeal gap) ${ }^{1}$.

When functional, the bulb favors the correction of VPI related obligatory speech errors. Sometimes physical correction alone is not enough and speech therapy is needed to establishing adequate velopharyngeal functioning during speech production. The combination of behavioral and prosthetic treatment allows for simultaneous correction of the insufficiency and incompetency resulting from mislearning. It is also an excellent alternative to optimize a future surgical correction of the insufficiency ${ }^{7}$. That is, once velopharyngeal sufficiency is established with the pharyngeal bulb, the speech pathologist can use behavioral strategies along with bulb reduction to modify the pattern of functioning of the velopharynx improving excursion of velum and pharyngeal walls affecting surgical recomendation. This intervention requires inter and even transdisciplinary work between areas of dentistry and speech pathology. It may be the only alternative available to patients who have a poor prognosis for surgical correction of insufficiency due to the presence of incompetency resulting from learned velopharyngeal inadequacy, such as hypodynamic velopharynx.

\section{OBJECTIVE}

The objective this study was to describe the process of intensive speech therapy for a 6 year old child using compensatory articulations while presenting with velopharyngeal insufficiency (VPI) and a history of cleft lip and palate.

\section{MATERIAL AND METHODS}

\section{Participant}

The subject for the speech program was a girl with a history of unilateral cleft lip and palate who presented classic signs of VPI after primary palatal surgery. The girl started treatment at a craniofacial center receiving a primary lip procedure at 6 months of age with Spina technique and a primary palatal procedure at 9 months of age with Von Langenback technique. Due to a wide cleft and lack of tissue to completely close the cleft without tension, a secondary correction was performed to repair a palatal fistula when the child was 2 years and 3 months old.

During the evaluation performed before the speech therapy the child presented with hypernasal speech and used compensatory articulations which compromised speech intelligibility. She was referred for speech therapy in her hometown, but no change was observed in her speech with the local interventions. At 6 years of age, a nasoendoscopic examination confirmed presence of VPI and learned incompetency, revealing a large gap associated with limited movement of pharyngeal walls and limited velar elevation characterizing a condition known as hypodynamic velopharyn $x^{14}$. With a bad prognosis for surgical correction of the insufficiency, the combined behavioral and prosthetic treatment was recommended for this case.

\section{Assessment procedures}

Before adapting the pharyngeal obturator, experienced speech pathologists performed an auditory-perceptual evaluation, nasometric assessment, along with nasoendoscopic and videofluorosocopic assessment of velopharyngeal function during speech. They also used aerodynamic assessment (PERCI) to estimate the velopharyngeal area during production of $/ \mathrm{p} /$. All assessments were repeated after the 60 sessions of speech therapy and adaptation of the pharyngeal obturator. 
The auditory perceptual assessment of speech was conducted during conversational speech for identification of presence or absence of hypernasality. Articulation was evaluated during repetition of words and phrases, with the speech pathologist identifying presence or absence of the use of compensatory articulation and phonological errors not expected for the child's age. Using nasometric assessment, a speech pathologist calculated the nasalance score to quantify the relative amount of nasal acoustic energy during oral speech production. A maximum nasalance score of $27 \%$ was expected during production of oral speech samples in Brazilian Portuguese language. Values above $27 \%$ were interpreted as indicative of hypernasality ${ }^{12}$.

The pressure-flow technique (modified rhinomanometry) was performed, as recommended by Warren and Dubois ${ }^{13}$ (1964) using a PERCI-SARS computerized system. The minimum cross-sectional area of the velopharynx (velopharyngeal area) was determined during the production of the plosive /p/ in the Portuguese word "papa." Areas of 0 to 4.9 $\mathrm{mm}^{2}$ were interpreted as representative of adequate velopharyngeal closure, areas of 5 to $9.9 \mathrm{~mm} 2$ were interpreted as representative of borderline but adequate velopharyngeal closure, and areas of 10 to $19.9 \mathrm{~mm}^{2}$ were interpreted as representative of borderline and inadequate velopharyngeal closure, and areas of $20 \mathrm{~mm}^{2}$ or more were interpreted as representative of inadequate velopharyngeal closure, according to the classification described by Warren ${ }^{14}$ (1997).

Nasoendoscopic and videofluoroscopic evaluations were performed for visualization of the velopharyngeal structures during speech production, allowing for verification of the ability of the velum to elevate and ability of the pharyngeal wall to move towards midline of the velopharynx. During both exams, the presence, pattern and size of the velopharyngeal gap were observed.

\section{Prosthetic treatment of VPI}

Once favorable dental conditions to support a speech obturator were observed, a palatal prosthesis was constructed by a dentist following 3 phases, each corresponding to one visit to the rehabilitation center. Phase one involved the development of the anterior portion of the device involving an acrylic plate with retention clips. The intermediate portion was added followed by. A speech bulb (pharyngeal obturator) that was shaped by a dentist along with the speech pathologist during production of oral sounds that favor adequate velopharyngeal functioning. The bulb was molded in thermoplastic material and later acrylized and polished, respecting the gap shape and size (Figures 1 and 2).

The three phases for development of the prosthesis took approximately 6 months, involving 3 visits of 4 days each to the center. Each visit was to construct the 3 portions of the device, with 2 months intervals between visits. The interval between each phase was needed to allow for the patient's adaptation to the device, which initially caused gagging and discomfort. Once the pharyngeal obturator was completed and the patient used the obturator daily and consistently, the speech therapy was initiated.

\section{Behavioral treatment of VPD}

An intensive speech therapy program was conducted at the craniofacial center, consisting of 3 phases with duration of 2 weeks each, involving speech therapy twice daily. Sessions lasted 50 minutes and initially targeted the elimination of compensatory articulations.

The first two phases targeted adequate production of oral place of articulation of high pressure speech sounds. Strategies involved manipulation of oral and nasal air pressure and airflow while establishing adequate place and manner of speech sounds as proposed by Golding-Kushner ${ }^{2}$ (2001) and Bzoch ${ }^{1}$ (2004). Visual, auditory and tactile feedback was used to contrast the target to the inadequate productions. The target was initially established in isolation followed by syllable production. Once the

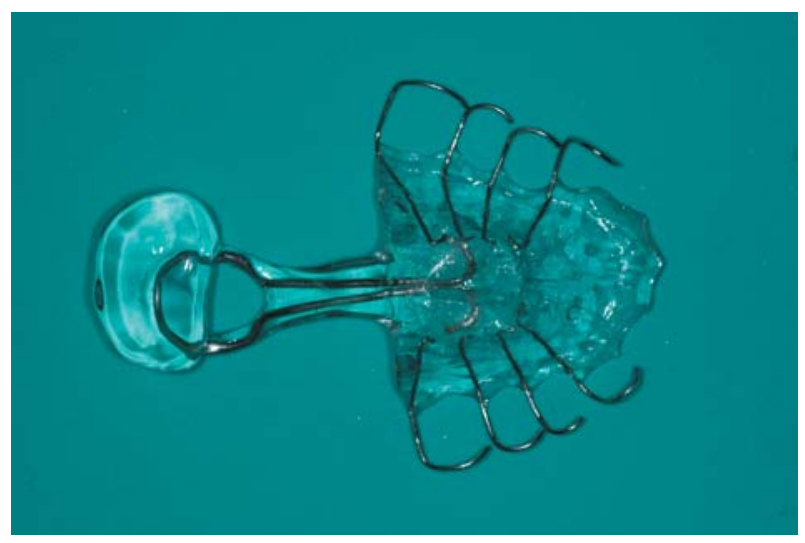

Figure 1- Pharyngeal obturator (speech bulb)

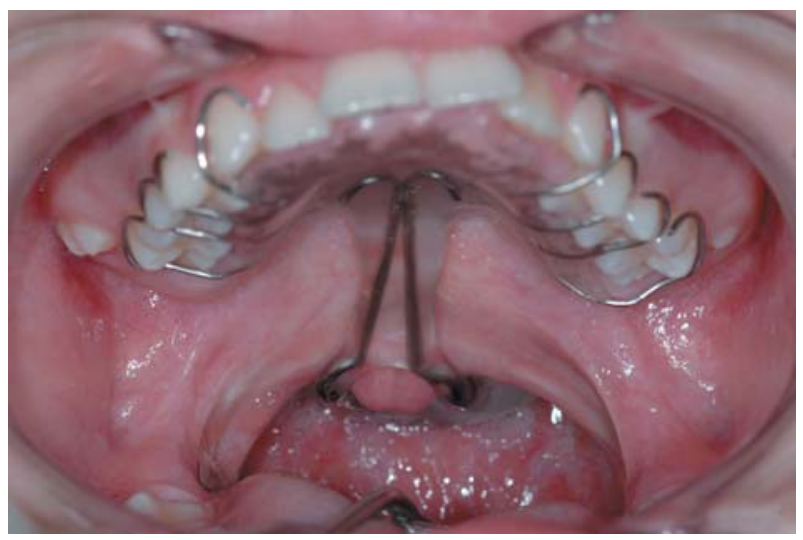

Figure 2- Intraoral speech bulb 
target sound was consistently produced adequately in syllables, words combining the target sound to liquid and nasal phonemes were introduced.

More than one target was introduced in the same word or phrase only after all targets were consistently produced with correct place of production. Later phrases were introduced and complexity was increased until targets were adequately produced during conversational speech.

The program involved orientations and training of the family and the child to monitor and to contrast adequate (target) and inadequate (CA) productions. With this program, all sounds trained during therapy (the new speech) were also practiced daily at home and monitored by the family between each phase of the program. The intervals between phases were needed, since the child could only come to the center during school vacation for periods no longer than 2 weeks each and because no speech pathologist was found in the child's hometown who could continue the work established during intensive therapy.

After the first 2 phases of work, we verified consistent use of oral place of production for all targets. Therefore, the third phase was initiated addressing velopharyngeal (VP) functioning. Perception of oral and nasal air pressure and airflow were addressed using auditory, visual and tactile feedback involving a plastic tube for auditory enhancement of oral and nasal air flow and "seeescape" and nasometry for visual enhancement of oral and nasal air flow. Once velopharyngeal closure was systematically obtained during oral samples, the contrast of oral and nasal was introduced addressing the ability to alternate between VP closure and VP opening. Once consistent adequate velopharyngeal functioning was established with the use of the pharyngeal obturator, a program for bulb reduction was initiated.

\section{Speech bulb reduction program}

This phase involved a partnership between the dentist and the speech pathologist. The prosthesis of the patient was replicated and the dentist was asked to shave off about $1 \mathrm{~mm}$ of acrylic around the surface creating a second bulb slightly smaller than the original. The removal of the acrylic was done using a bur and followed with finishing and final polishing. Once the device with the reduced bulb size was finished, speech therapy resumed, contrasting oral production with the original device to that with the smaller bulb. Feedback of oral and nasal air pressure and airflow were provided. Once productions were similar with both bulbs, the dentist was asked again to remove the acrylic of the larger bulb until it was about $1 \mathrm{~mm}$ smaller than the reduced bulb.

The reductions were repeated until the maximum displacement of velopharyngeal structures was established and verified by the lack of ability of the child to avoid nasal air leak. About $6 \mathrm{~mm}$ of acrylic was removed from the bulb surface once the reduction program was finished (Figures 3, 4, 5 and 6).

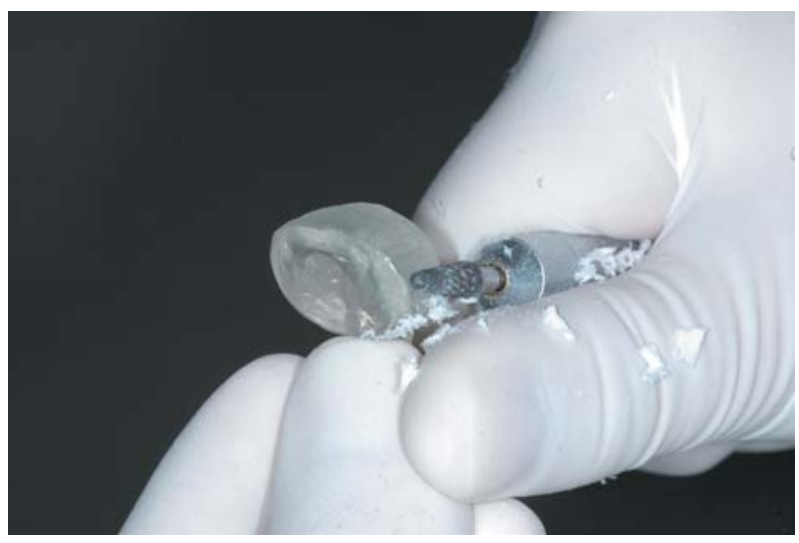

Figure 3- Pharyngeal obturator reduction with bur - phase of fray

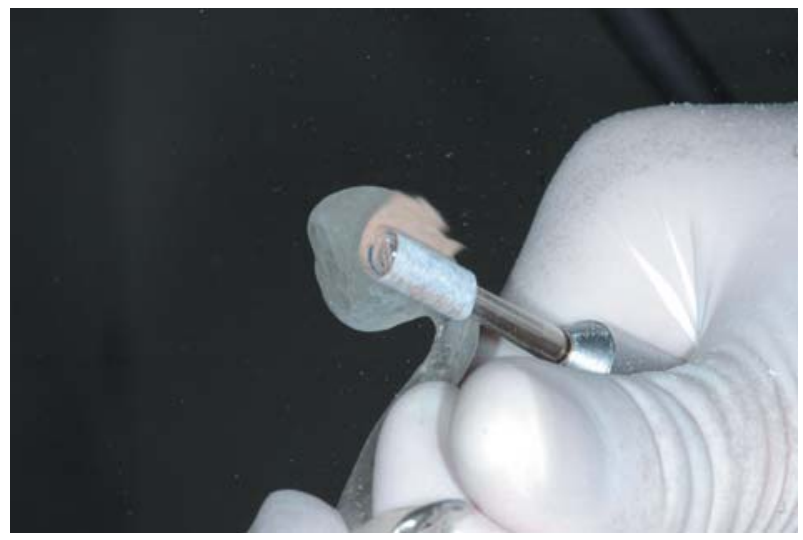

Figure 4- Pharyngeal obturator reduction with sandy paper - phase of finishing

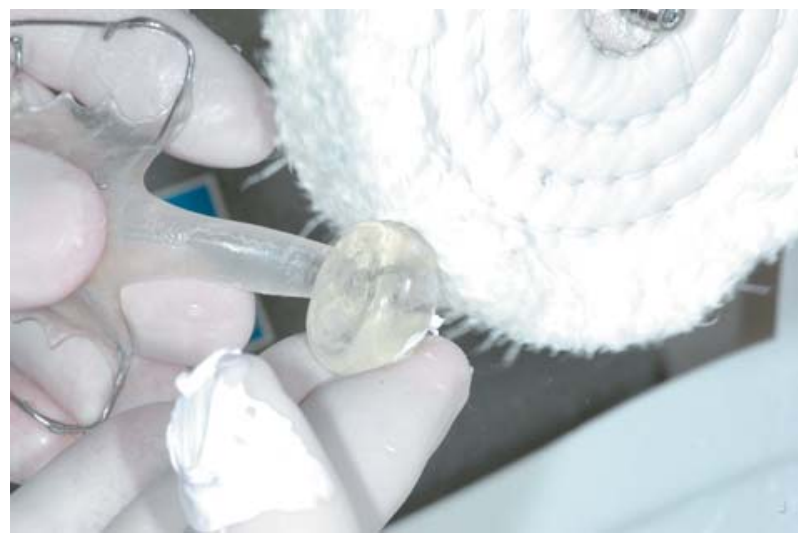

Figure 5- Pharyngeal obturator reduction with grinding paste - phase of polishing 


\section{RESULTS}

A total of 60 direct speech sessions were completed during the 3 phases of the program. The assessment procedures described above were performed before and after the intensive speech therapy and bulb reduction program. As presented and described in Figure 7 all speech alterations present before the interventions were absent after the program (Figure 7).

\section{DISCUSSION}

The role of the primary surgeries in cases of CLP is to correct the cleft without compromising facial growth while establishing an adequate structure to support oral functions like feeding and speech. Literature indicates that between 5\% and 50\% of the cases who receive primary palatoplasty to

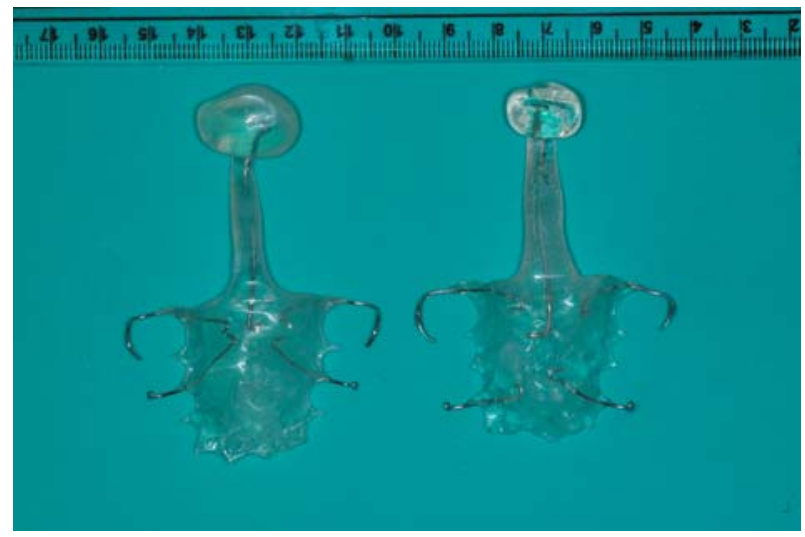

Figure 6- Speech bulb original X Speech bulb with reduction correct cleft palate (average of $20 \%$ ) still present VPD after primary repair due to insufficiency, incompetency or both. Correction of VPD can involve a physical (surgery or prosthesis) and/or behavioral treatment ${ }^{4,5,9,15}$. In some cases the management of VPD with speech therapy alone (in cases of incompetency) or with secondary surgery (in cases of insufficiency) do not have good prognosis requiring the use of prosthetic management. The child studied was referred for a treatment program which allowed for the simultaneous management of the insufficiency and incompetency involving behavioral manipulation of the velopharyngeal mechanism.

According to Pinto, Dalben and Pegoraro-Krook ${ }^{8}$ (2007), prosthetic treatment of VPI involves the use of pharyngeal obturators (speech bulbs) and the outcome of this approach depends on the degree of hypernasality, type of compensatory articulation used, age and dental conditions of the candidate, along with structural and functional conditions of the palate and pharyngeal walls. The patient described in this case report fitted the criteria for recommendation of the combined prosthetic and behavioral treatment. In this case, the use of a surgical procedure for velar lengthening would not resolve the insufficiency since the VP gap was too large. The results of a pharyngeal flap procedure was obscure due to limited movement of pharyngeal walls. The use of a pharyngeal obturator combined with a speech therapy program was recommended to rehabilitate this patient's speech and to optimize a later surgical treatment to correct the insufficiency.

This case report shows evidence that a pharyngeal obturator can be used as a temporary, conservative, and easily reversible treatment of VPD postponing

\begin{tabular}{|c|c|c|}
\hline Assessment & BEFORE Intervention & AFTER Intervention \\
\hline Auditory perceptual assessment of resonance & Hypernasality & Normal \\
\hline $\begin{array}{l}\text { Auditory perceptual assessment of } \\
\text { articulation }\end{array}$ & $\begin{array}{l}\text { Presence of compensatory } \\
\text { articulation - glottal stops }\end{array}$ & $\begin{array}{l}\text { Use of correct oral place of } \\
\text { articulation }\end{array}$ \\
\hline $\begin{array}{c}\begin{array}{c}\text { Nasometry }- \text { oral sample }(27 \% \text { or less }= \\
\text { normal })\end{array}\end{array}$ & $\begin{array}{c}37 \% \\
\text { Indicative of hypernasality }\end{array}$ & $\begin{array}{l}23 \% \\
\text { Indicative of adequate } \\
\text { resonance }\end{array}$ \\
\hline $\begin{array}{l}\text { Aerodynamic assessment velopharyngeal } \\
\text { area /papa/ }\end{array}$ & $\begin{array}{l}29 \mathrm{~mm}^{2} \\
\text { Indicative of VPI }\end{array}$ & $\begin{array}{c}0.08 \mathrm{~mm}^{2} \\
\text { Indicative of borderline closure }\end{array}$ \\
\hline Nasoendoscopic assessment & $\begin{array}{c}\text { Large VP gap } \\
\text { Limited movement of pharyngeal } \\
\text { walls and velum }\end{array}$ & $\begin{array}{c}\text { Adequate VP closure at } \\
\text { the speech bulb with good } \\
\text { displacement of pharyngeal } \\
\text { walls }\end{array}$ \\
\hline Videofluoroscopic assessment & $\begin{array}{c}\text { Large VP Gap } \\
\text { Limited velar elevation and limited } \\
\text { wall displacement }\end{array}$ & $\begin{array}{l}\text { Touch of the velum and } \\
\text { pharyngeal wall to the bulb }\end{array}$ \\
\hline Speech intelligibility & Severely compromised & Normal \\
\hline
\end{tabular}

Figure 7- Results of speech and velopharyngeal function assessment before and after intensive therapy and bulb reduction 
and optimizing future surgical treatment. The device can be easily individualized according to the anatomical and functional needs of the patient. The functional adaptation of a pharyngeal obturator, however, requires teamwork between the dentist and the speech pathologist as well as the family and the patient.

If, on one hand, there are many advantages for the use of a pharyngeal obturator, on the other hand there are also disadvantages, all of which need to be considered before recommending the procedure. The treatment itself is usually planned in 3 phases, each requiring one visit to the craniofacial center one, for the construction of each portion of the palatal prosthesis. The number of visits, however, can increase when there are difficulties with the adaptation of the device. Acceptance and understanding by the family and patient of the type of treatment and the burden it entails are essential for its success. The device will occupy the velopharyngeal space (the gap area), leading to discomfort and gagging at the beginning ${ }^{8}$. Different than a surgery, for which the patient is anesthetized, with the palatal prosthesis the patient must be compliant during all phases of the treatment, particularly during functional adaptation and shaping of the speech bulb which requires speech production. In the present case both the patient and the family were compliant to the treatment prescribed. Furthermore the therapy addressing the use of adequate place of sound production involved daily practice at home, requiring the compliance and commitment of the child and her family. Orientation and training of the family to monitor the exercises at home were needed for teaching discrimination between the old and the new speech.

The bulb reduction program, in particular, is described in the literature as an alternative to elicit or increase movement of the pharyngeal walls, allowing for a reduction of the VP gap, thus optimizing a future surgical correction of a velopharyngeal insufficiency and fostering the use of a surgical procedure which entails a lower risk of leading to respiratory obstruction ${ }^{10,16}$. For some patients, like the one in this case report, the presence of learning errors regarding velopharyngeal functioning can lead to unsuccessful surgical results independent of the surgical procedure used, particularly due to velopharyngeal hypodynamism. In such cases the behavioral therapy combined with prosthetic treatment is an excellent alternative to establish adequate velopharyngeal function for speech.

\section{Final Consideration}

Patients with speech disorders associated to VPD can be successfully treated with behavioral speech therapy combined with a prosthetic approach guided to improve speech intelligibility and to optimize future surgical correction of VP insufficiency.

\section{REFERENCES}

1- Bzoch KR. Communicative disorders related to cleft lip and palate. Austin: Pro-Ed; 2004.

2- Golding-Kushner KJ. Therapy techniques for cleft palate speech and related disorders. San Diego: Singular-Thompson Learning; 2001.

3- Kuehn DP, Moller KT. Speech and language issues in the cleft palate population: the state of the art. Cleft Palate Craniofac J. 2000;37:1-35

4- Mazaheri M. Indications and contraindications for prosthetic speech appliances in cleft palate. Plast Reconstr Surg Transplant Bull. 1962;30:663-9.

5- Park S, Saso Y, Ito O, Tokioka K, Takato T, Kato K, et al. The outcome of long-term follow-up after palatoplasty. Plast Reconstr Surg. 2000;105:12-7.

6- Peterson-Falzone SJ, Hardin-Jones MA, Karnell MP. Cleft palate speech. Saint Louis: Mosby; 2001.

7- Peterson-Falzone SJ, Hardin-Jones MA, Karnell MP. Communication disorders associated with cleft palate. In: Peterson-Falzone SJ, Hardin-Jones MA, Karnell MP. Cleft palate speech. Saint Louis: Mosby; 2001. p. 162-98.

8- Pinto JHN, Dalben GS, Pegoraro-Krook MI. Speech Intelligibility of patients with cleft lip and palate after placement of speech prosthesis. Cleft Palate Craniof J. 2007;44:635-41.

9- Pinto JH, Pegoraro-Krook MI. Evaluation of palatal prosthesis for the treatment of velopharyngeal dysfunction. J Appl Oral Sci. 2003; 11:192-7.

10- Shelton RL, Lindquist AR, Arndt WB, Elbert M, Youngstrom KA. Effect of speech bulb reduction of movement of the posterior wall of the pharynx and posture of tongue. Cleft Palate J. 1971;8:10-7. 11- Spina V, Psillakis JM, Lapa FS. Classificação das fissuras lábio-palatinas: sugestão de modificação. Rev Hosp Clin Fac Med S Paulo. 1972;27: 5-6.

12- Trindade IEK, Genaro KF, Dalston RM. Nasalance scores of normal brazilian portuguese speakers. Braz J Dysmorphol Speech Hear Disord. 1997;1:23-34.

13- Warren DW, Dubois AB. A pressure-flow technique for measuring velopharygeal orifice area during continuous speech. Cleft Palate J. 1964;16:52-71.

14- Warren DW, Rouget AH, Hinton VA. Aerodynamics. In: McNeil $M$, ed. Clinical management of sensorimotor speech disorders. New York: Thieme Medical Publishers; 1997.

15- Witt PD, Marsh JL, Marty-Grames L, Muntz HR, Gay WD. Management of the hypodynamic velopharynx. Cleft Palate Craniofac J. 1995;32:179-87.

16- Witt PD, Rozelle AA, Marsh JL, Marty-Grames L, Muntz HR, Gay WD, et al. Do palatal lift prostheses stimulate velopharyngeal neuromuscular activity? Cleft Palate Craniofac J. 1995;32:469-75. 